\title{
130. Einfluss des Ikterus auf die Entwicklung von Schistosoma japonicum im Wirtskörper.
}

\author{
Von Tarô Sonoda.
}

(Comm. by A. FuJinami, M.I.A., Oct. 12, 1933.)

Es ist schon bekannt, dass die Cercarien von Schistosoma japonicum sehr leicht in die Haut aller Säugetierarten eindringen, die dadurch an Schistosomiasis leiden, und dass solche Hautinvasion auch ohne Schwierigkeiten bei den natürlich-immunen Tieren, z. B. bei den Hühnern, geschieht. Obwohl die Galle gegenüber den Cercarien einen giftigen Stoff darstellt, kann eine beträchtliche Schistosomiasis nach der stattgefundenen Hautinvasion der Cercarien auch bei den ikterischen Kaninchen hervorgerufen werden, wie Arima neuerdings erwiesen hat. Durch eine andere zufällige Beobachtung Arimas angeregt, hat sich Verfasser entschlossen, eine eingehende, systematische Untersuchung über das Verhalten der Schistosoma-Invasion gegenüber dem Ikterus beim Kaninchen vorzunehmen. Er legte sich die Fragen vor: 1) Ist es doch möglich, dass unter gewissen Bedingungen künstliche Immunität gegenüber der Schistosomiasis durch den Ikterus hervorgerufen wird? Mit anderen Worten, können beim Experimente wirklich Fälle vorkommen, bei denen die in die Haut eingedrungenen Cercarien sämtlich innerhalb des ikterischen Wirtskörpers zu grunde gehen, so dass dieser sich schliesslich von der Schistosomiasis befreit? 2) Auf welche Weise geht die Vernichtung der Cercarien vor sich, vorausgesetzt, dass diese innerhalb des Wirtskörpers überhaupt möglich ist?

Operativer Weise wurde der Ductus choledochus bei zahlreichen Kaninchen unterbunden, um einen Stauungsikterus hervorzurufen. Nachdem sich dann in verschieden langen Zeiträumen nach dem Auftreten des Ikterus die Cercaria-Invasion an der ikterischen Haut der Bauchoder Inguinalgegend vollzogen hat, wird das Schicksal der eingedrungenen Cercarien im Wirtskörper vorsichtig weiter verfolgt.

Nach den Untersuchungsergebnissen des Verfassers sind tatsächlich die Würmer im stande, bis zu einem gewissen Grade dem Ikterus gegenüber Widerstand zu leisten, so dass die Würmer im ikterischen Wirt gut gedeihen und sich weiter entwickeln. Bei Experimenten aber, die in einem anderen Ikterusstadium ausgeführt worden waren, hatte sich keine Schistosomiasis entwickelt, da die eingedrungenen Cercarien sämtlich im ikterischen Wirtskörper zu grunde gegangen waren. Woher 
kommt dieser Unterschied? Wann, wie, wo und warum sterben die Eindringlinge? Um auf diese Fragen eine Antwort zu geben, erlaubt sich Verfasser, die Ergebnisse seiner Experimente beim Kaninchen, kurz zusammengefasst, anzugeben, wie folgt.

\section{1) Die Invasionsgeschwindigkeit der Cercarien.}

An der ikterischen Haut geschieht die Invasion der Cercarien etwas langsamer, als an der gesunden. Die Gewebeveränderungen entzündlicher Natur, die gewissermassen als Abwehrerscheinung zu deuten sind, treten dabei etwas langsamer und geringeren Grades auf, wie sonst.

Von den aus zahlreichen Experimenten gewonnen Ergebnissen des Verfassers sei hier nur folgendes erwähnt. Während an der gesunden Haut das Eindringen der Cercarien schon im etwa 2 Minuten beginnt und das Passieren des Hautgewebes von sämtlichen Würmchen ungefähr in 3 Tagen beendet ist, vollzieht sich dieser Vorgang an der ikterischen Haut etwas langsamer: In einem früheren Stadium des Ikterus (z.B. am 4. Tage nach der Choledochus-Unterbindung) beginnt die Cercaria-Invasion in etwa 5 Minuten, und in ungefähr 4 Tagen haben sämtliche Cercarien die Haut passiert. In einem noch etwas späteren Stadium des Ikterus (z.B. am 10. Tage nach der Choledochus-Unterbindung) wurde dagegen die erste Cercaria-Invasion ins Hautgewebe erst in 7 Minuten beobachtet. In solchen Fällen wurden aber später im Hautgewebe viel Würmchen tot gefunden.

Was die durch die Cercaria-Invasion hervorgerufene reaktiventzündliche Zellinfiltration des Hautgewebes betrifft, so beginnt sie an der normalen Haut schon 30 Minuten nach der ersten Cercaria-Invasion, erreicht ihren Höhepunkt in 1-2 Tagen und verschwindet ungefähr in 5 Tagen. Bei der in einem früheren Stadium des Ikterus - z.B. 4 Tage nach der Choledochus-Unterbindung - stattfindenden Invasion tritt die entzündliche Zellinfiltration erst 1 Stunde nach der Cercaria-Invasion auf und ist am ausgeprägtesten nach 1-3 Tagen; etwa in 7 Tagen nach der Hautapplikation der Cercarien ist sie zu Ende. In den Fällen, bei denen die Cercaria-Invasion in einem noch späteren Ikterusstadium (z.B. 10 Tage nach der Choledochus-Unterbindung) stattfindet, tritt die entzündliche Zellinfiltration in etwa 2 Stunden auf und bleibt so lange, bis die da abgestorbenen Cercarienkörper vollständig zu grunde gegangen sind. 


\section{2) Das Schicksal der eingedrungenen Würmer im Wirtskörper.}

Falls die Invasion der Cercarien in die Haut vor oder an dem 5. Tage nach der Choledochus-Unterbindung geschieht, können diese im Wirtskörper beinahe zu gewöhnlicher Entwicklung gelangen. Dagegen kommen die am 10.-20. Tage nach der Gallengangunterbindung invasierten Würmer schliesslich gar nicht zur Entwicklung. Die Grenze zwischen den positiven Resultaten und den negativen in bezug auf die Würmerentwicklung fällt ungefähr auf solche Fälle, bei denen die Hautinvasion der Cercarien am 6.-7. Tage nach der Choledochus-Unterbindung geschah. Selbstverständlich variiert dieses Verhalten einigermassen je nach der Körperkonstitution des Wirtstieres sowie der Vitalität der Cercarien.

Es gab solche Fälle, bei denen die Cercaria-Invasion am 6. oder 7. Ikterustage geschah und der Sterbeort der Eindringlinge ausschliesslich die Lunge war, während in anderen Fällen von demselben Datum nur eine gewisse Zahl Cercarien oder weniger entwickelter Würmer in dem Lungengewebe tot gefunden wurden.

\section{3) Histologische Befunde des Todesortes der eingedrungenen Cercarien.}

Bei den „immun" gemachten Kaninchen, wovon oben die Rede war, stirbt ein Teil der Cercarien schon im Hautgewebe ab, während eine grössere Anzahl in die Lunge gelangt, um dort innerhalb der Blutkapillaren zu grunde zu gehen. In den anderen Körperteilen wird dagegen nirgends ein totes Würmchen gefunden.

Im Hautgewebe finden sich abgestorbene Cercarien, von denen einige schon veraltet aussehen, von entzündlich-infiltrierten Zellen umgeben. Solche toten Cercaria-Kadaver beobachtet man meistens an den oberflächlichen Hautschichten, wie es oft bei der Haut der natürlichimmunen Tiere (z.B. Hühner, Enten) der Fall ist. Bei der letzteren aber tritt die reaktiv-entzündliche Gewebeveränderung ausgeprägter auf. Es fehlt zwar nicht an abgestorbenen Cercarien, die noch nicht von einer Zellinfiltration umgeben sind. Solche Cercarien aber werden vorwiegend an den tieferen Bindegewebeschichten der Haut gefunden und nicht selten sind ihre Kernmassen einer starken Zusammenschrumpfung oder Hyperchromasie anheimgefallen. In der Lunge werden histologisch $\mathrm{ab}$ und $\mathrm{zu}$ kleine Tuberkel nachgewiesen, die tote oder allmählich $\mathrm{zu}$ grunde gehende Würmer enthalten. 
Man darf wohl annehmen, dass das Absterben der eingedrungenen intravasculären Cercarien durch eine rechtzeitige Einwirkung der aus der Galle herstammenden giftigen Substanzen stattgefunden hat, wobei die pathologisch veränderte Körperbeschaffenheit des Wirtes auch in Betracht kommen könnte. 